\title{
Model predictive for Mobile robot control
}

\author{
Alireza Rezaee
}

\begin{abstract}
This paper proposes a Model Predictive Controller (MPC) for control of a P2AT mobile robot. MPC refers to a group of controllers that employ a distinctly identical model of process to predict its future behavior over an extended prediction horizon. The design of a MPC is formulated as an optimal control problem. Then this problem is considered as linear quadratic equation (LQR) and is solved by making use of Ricatti equation. To show the effectiveness of the proposed method this controller is implemented on a real robot. The comparison between a PID controller, adaptive controller, and the MPC illustrates advantage of the designed controller and its ability for exact control of the robot on a specified guide path.
\end{abstract}

Index Terms - robot, control, model, prediction.

\section{INTRODUCTION}

$\mathrm{M}$ odel Predictive Controllers (MPC) are widely adopted

in industry as effective tools for dealing with large scale multivariable and multi-constrained control problems (Guang et al,2005; Camacho and Bordons, 1999; Nagy et al, 2005). The main idea of MPC lies in online construction of the system model, predicting its future states and generating the required control actions by repetitive solution of an optimal control problem. Issues may arise for guaranteeing closed-loop stability, model uncertainty handling and reducing the on-line computations.

There are three kinds of MPC controller schemes that use different methods for system modeling but are similar to each other in control process (Likar et al, 2007) :

1- MAC: uses impulse response for system modeling,

2- GPC : uses transfer function for system modeling,

3- DMC: uses step response for system modeling.

These controllers optimize a cost function that depends on the control law (Hauge et al, 2002).

Although DMC is primarily developed for control of chemical processes (Camacho and Bordons, 1999; Garcia et al., 1989; Limon et al, 2005), it has been extended

Assistant Professor of Department of system and Mechatronics Engineering, Faculty of New Sciences and Technologies, University of Tehran, Tehran, Iran, arrezaee@ut.ac.ir. successfully to other applications such as motorway traffic systems (Bellemans et al, 2006), switching max-plus-linear discrete event systems and simplified model of biped walking models(van et al, 2006; Azevedo et al, 2002) . However implementation of this control scheme in robotics has been less reported and seems to be in still in its infancy (Limon et al, 2005; Kouvaritakis et al, 2006). In this work we concentrate on application of MPC/DMC controllers in position control of robotic systems.

The rest of this paper is organized as follows: The next section presents the idea of MPC controller, section 3 describes P2AT robot, section 4 discusses the effect of variation of DMC parameters on speed error, section 5 discusses results of the experimental implementation of MPC/DMC on a real robot, and finally the last section contains the conclusions.

\section{MODEL PREDICTIVE CONTROLLER}

The main strategy of a model predictive controller is illustrated in Figure 1. In a typical MPC algorithm the system outputs are predicted for a certain interval of time (prediction horizon) by making use of a proper system model which is constructed based on the information (inputs and outputs) gathered from the system past as well as future control signals that have to be determined properly. As shown in the figure the control signal is a sequence of step functions with variable amplitude. Amplitudes of these inputs are obtained by solving an optimization problem that tries to keep the system output close to the reference set point. Objective function of this problem is usually a quadratic function of the difference between the predicted output signals and the reference trajectory.

All the MPC algorithms using a linear model have similar behavior. Here we demonstrate how DMC works.

$$
Y=A \Delta u+Y_{0}
$$

Where A includes the step response, $\mathrm{Y}$ is the predicted output, Y0 is past output, and $\mathrm{u}$ is the control law (Azevedo et al, 2002, Shridhar and Cooper, 1997). 


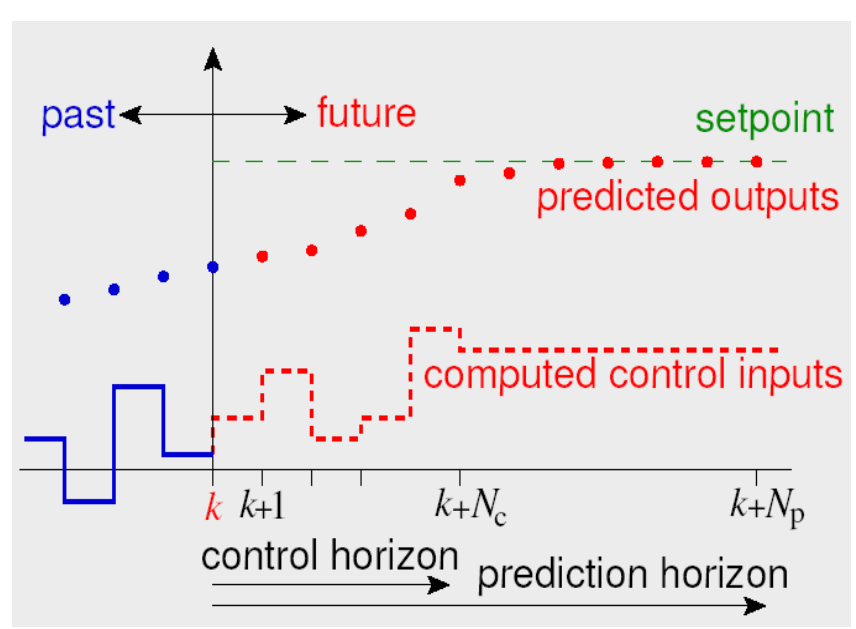

Fig.1. Methodology of MPC.

Due to uncertainities of the model it is very hard to achieve the exact value of A to satisfy the desired bahavior. To compansate for this problem an error term is added to the system output:

$$
\begin{aligned}
& \text { correction }=y_{n}^{p}-y_{n} \\
& \hat{Y}=A \Delta u+Y_{0}+\left[\begin{array}{l}
y_{1}^{p}-y_{1} \\
\cdots \\
y_{n}^{p}-y_{n}
\end{array}\right]=A \Delta u+Y_{0}+E_{f}
\end{aligned}
$$

Where the correction term represents the difference between the current plant actual output and the output extracted from the model. The error vector over prediction horizon is then written as

$$
E=R-\hat{Y}=R-A \Delta u-Y_{0}-E_{0}
$$

Using the above expression a quadratic cost function, J, can be defined which is minimized to obtain the optimal controller

$$
\min _{\Delta u} J=E^{T} W_{1} E+\Delta u^{T} W_{2} \Delta u
$$

where W1 and W2 are constants. The modified control law is obtained as:

$$
\Delta u=\left(A^{T} W_{1} A+W_{2}\right)^{-1} A^{T} W_{1} E^{\prime} \equiv K_{c} E^{\prime}
$$

Fig. 2 shows the structure of a model predictive controller. In this configuration the block labeled as "Model" contains the model of the robot that predicts the behavior of the robot over a certain time horizon. The Future Inputs $(\mathrm{u}(\mathrm{t}+\mathrm{k} \mid \mathrm{t}))$ are calculated under constrains and by optimizing a cost function. This process continues until the end of the trajectory.

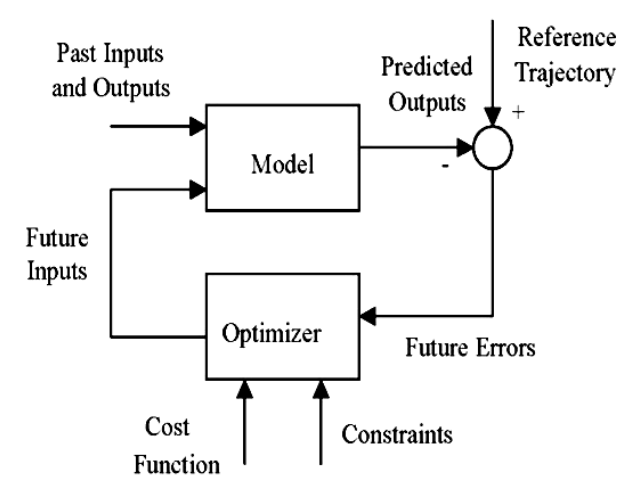

Fig. 2. The structure of a MPC.

\section{A. The Algorithm of a DMC controller}

The algorithm of a DMC controller is as follows:

1- Obtain model of the robot to be controlled.

2- Use the model to predict behavior of the robot over a certain time horizon.

3- Calculated the $\mathrm{E}$ from equation (4).

4- Determine the control action by optimizing a performance index, which typically is the error between the outputs predicted from the model and the desired output over the time horizon.

5- Apply the optimal control actions and then measure robot outputs over the time horizon. The measured values at the final stage will be used as initial conditions of the model in the next iteration.

6- Repeat steps 2 to 5 until the end of the trajectory.

\section{ROBOT CONTROL}

For robot control with MPC controller we need to have the model equation of the robot (Fig. 3). The robot under consideration in this study is a four wheel P2AT robot in which wheels of the robot are controlled independently.

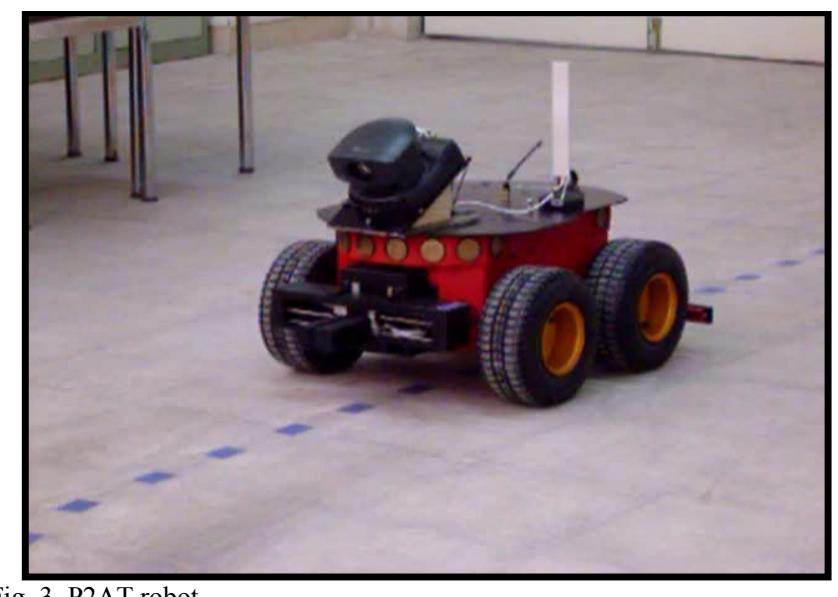

In order to obtain the system model and design the proper controller for it, a sound appreciation of dynamic behavior of 
the system is needed. To do that a simple sketch of the robot is shown if Fig. 4, it is assumed that the distance between each wheel is constant and four wheels have the same radius. Kinematic model of the robot is described by

$$
\begin{gathered}
\mathrm{x}(\mathrm{k}+1)=\left[\begin{array}{c}
\mathrm{x}(\mathrm{k}+1) \\
\mathrm{y}(\mathrm{k}+1) \\
\theta(\mathrm{k}+1)
\end{array}\right] \\
=\left[\begin{array}{c}
\mathrm{x}(\mathrm{k})+\vartheta(\mathrm{k}) \Delta \mathrm{t} \cos (\theta(\mathrm{k})) \cos (\alpha(\mathrm{k})) \\
\mathrm{y}(\mathrm{k})+\vartheta(\mathrm{k}) \Delta \mathrm{t} \sin (\theta(\mathrm{k})) \cos (\alpha(\mathrm{k})) \\
\theta(\mathrm{k})++\vartheta(\mathrm{k}) \Delta \mathrm{t} \sin (\alpha(\mathrm{k})) / \mathrm{L}
\end{array}\right]=\mathrm{F}[\mathrm{z}(\mathrm{k})]=\mathrm{F}[ \\
\left.\mathrm{x}_{\mathrm{m}}(\mathrm{k}), \mathrm{u}(\mathrm{k})\right] \\
S_{i}=\frac{(\dot{X}-a \cos \theta)}{\cos \theta}-\frac{L}{2} w \dot{\theta} \\
S_{o}=\frac{(\dot{X}-a \cos \theta)}{\cos \theta}+\frac{L}{2} w \dot{\theta}
\end{gathered}
$$

Where $\mathrm{u}(\mathrm{k})=[\vartheta(\mathrm{k}), \alpha(\mathrm{k})]^{\mathrm{T}}$ is the control vector for motion tracking, $\Delta \mathrm{t}$ is the sample period and $S_{i}$ and $S_{o}$ denote speed of the left and right wheels respectively and the distance between wheels is shown by L and w. Moreover, a is the distance between reference point of the robot and the wheels. Additionally, the position of the robot in global reference frame is specified by coordinates $\mathrm{X}$ and $\mathrm{Y}$. The angular difference between the global and local reference frames is given by $\theta$.

Such a non-linear system is open loop controllable, which can be linearised in order to use traditional linear feedback control to regulate the robot. But if the robot operates over a large range in its state space, especially when the robot turns around corners, the linearization of the kinematics will lead to the loss of controllability. Since the MPC's models are based on linear regressions.

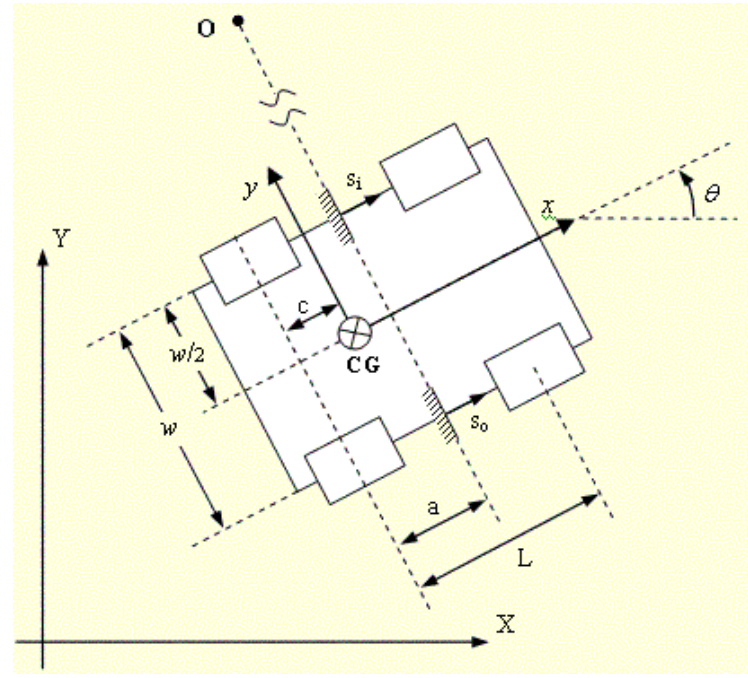

Fig. 4. Schematic of the P2AT robot.

\section{THE EFFECT OF DMC PARAMETERS ON CONTROLLER PERFORMANCE}

Due to the simple nature of the linear mathematical models (Mayne et al, 2000; Axehill, 2004; Axehila, 2004) most of the MPCs including impulse and step response models and the transfer function model are based on this type of model description (Dougherty and cooper, 2004; Gilbert and Tan., 1991). Thus, the first step in controller design is to linearize the model equations and then calculate the control laws. Figure 5 shows the schematic of the MPC controller that is connected to the system (robot). Rest of this paper is devoted to study effect of different parameters of the model on the controller performance. To do that a series of experiments were conducted on a simple straight path and speed of robot was measured for different instances (Figs. 6-13).

MPC controller is to optimize a cost index $\mathrm{J}(\mathrm{x}(\mathrm{k}), \mathrm{u}(\mathrm{k})))$ under the constraints of formula 7 :

$\min _{\mathrm{u}(\mathrm{k})} \mathrm{J}(\mathrm{x}(\mathrm{k}), \mathrm{u}(\mathrm{k}))$

The current control vector is chosen to minimise thee state errors and control energy over several steps in future so that the path tracking of the robot is smooth aand stable. Therfore, the cost index can be expressed as

$$
\underset{\mathrm{d}) \|^{2}}{\mathrm{~J}}=\frac{1}{2} \sum_{\mathrm{i}=\mathrm{N}_{1}}^{\mathrm{N}_{2}}\left\|\mathrm{x}_{\mathrm{r}}(\mathrm{k}+\mathrm{i})-\mathrm{x}(\mathrm{k}+\mathrm{i})\right\|^{2}+\frac{1}{2} \sum_{\mathrm{i}=1}^{\mathrm{N}_{\mu}} \lambda_{\mathrm{i}} \| \Delta \mathrm{u}(\mathrm{k}+\mathrm{i}-
$$

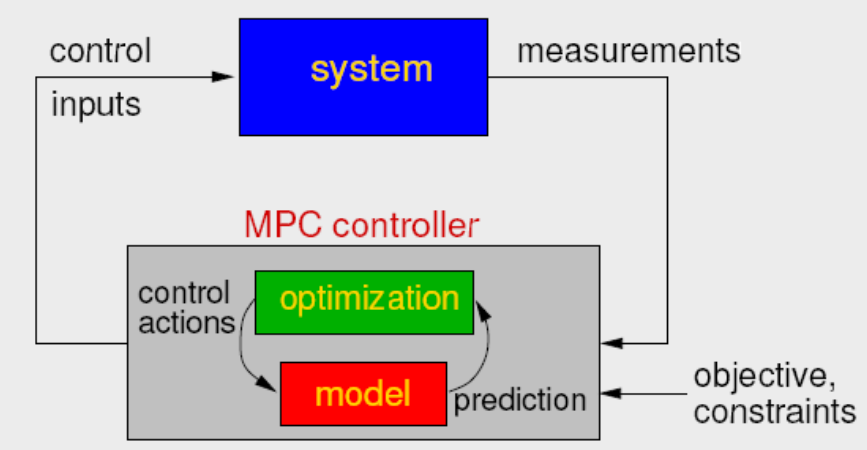

Fig. 5. Connection between MPC controller and the system (robot).

\section{A. Effect of $M$ (control horizon)}

Figures 6, 7 show the effect of control horizon parameter, $\mathrm{M}$, on the control output and control law respectively. It is observed that by increasing value of $M$ the settling time is decreased and the control effort is increased. This also increases the computational complexity. According to the experimental results the optimal value of $\mathrm{m}$ selected as 2 . 


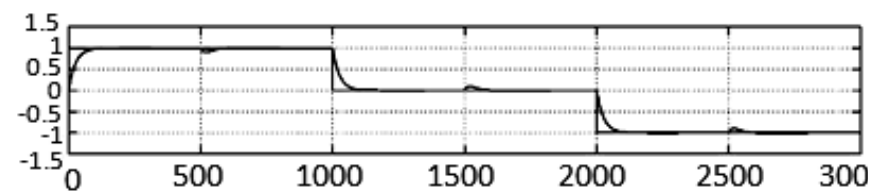

$\mathrm{M}=1, \mathrm{~N}=20, \mathrm{P}=5, \alpha=0.5, \mathrm{~W} 2=0$.

(a)

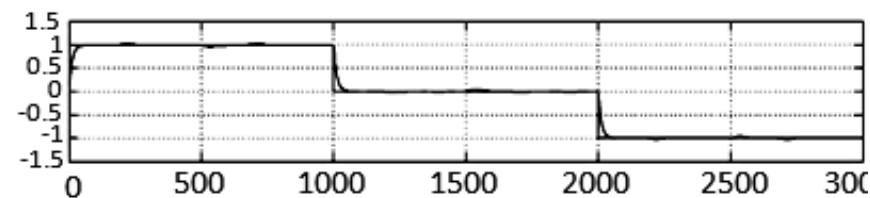

$\mathrm{M}=2, \mathrm{~N}=20, \mathrm{P}=5, \alpha=0.5, \mathrm{~W} 2=0$.

(b)

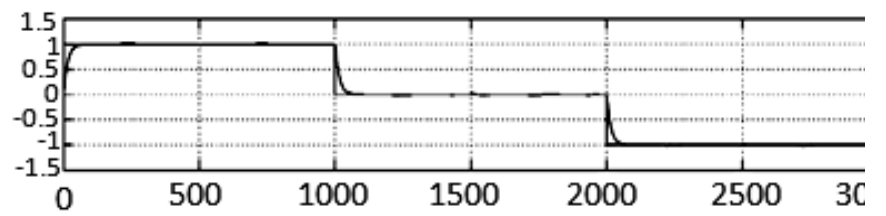

$\mathrm{M}=4, \mathrm{~N}=20, \mathrm{P}=5, \alpha=0.5, \mathrm{~W} 2=0$.

(c)

Fig .6. output.

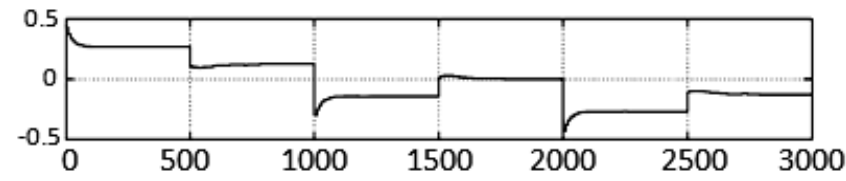

$\mathrm{M}=1, \mathrm{~N}=20, \mathrm{P}=5, \alpha=0.5, \mathrm{~W} 2=0$.

(a)

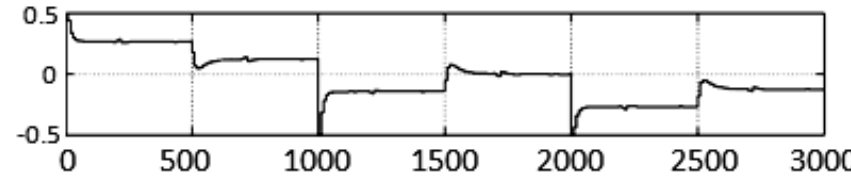

$\mathrm{M}=2, \mathrm{~N}=20, \mathrm{P}=5, \alpha=0.5, \mathrm{~W} 2=0$.

(b)

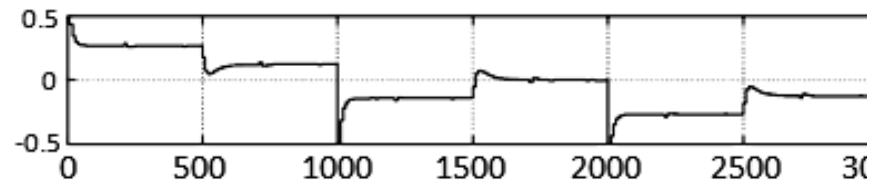

$\mathrm{M}=4, \mathrm{~N}=20, \mathrm{P}=5, \alpha=0.5, \mathrm{~W} 2=0$.

(c)

Fig.7.control law

\section{B. Effect of $P$ (prediction horizon)}

Figures 8, 9 show the system output and control law for two different values of parameter $P$ respectively. It can be seen that by increasing value of $\mathrm{P}$ the settling time is decreased and the control effort is increased and the computational complexity is increased simultaneously.

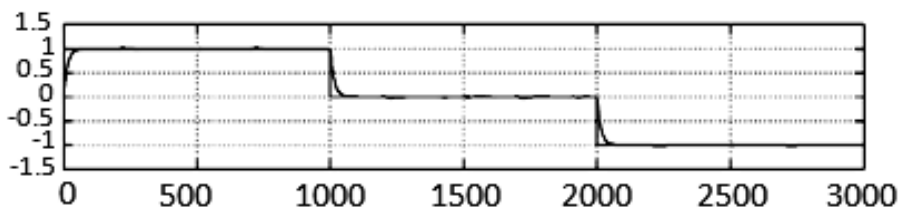

$\mathrm{M}=2, \mathrm{~N}=20, \mathrm{P}=3, \alpha=0.5, \mathrm{~W} 2=0$.

(a)

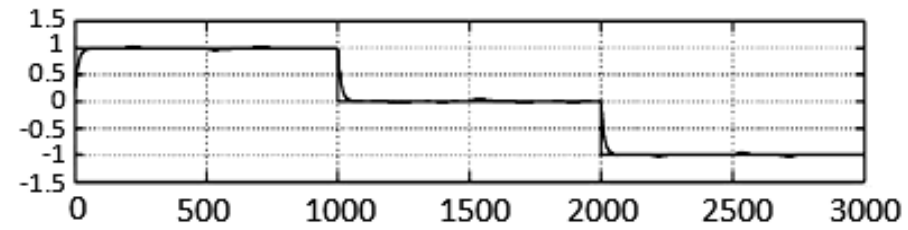

$\mathrm{M}=2, \mathrm{~N}=20, \mathrm{P}=10, \alpha=0.5, \mathrm{~W} 2=0$.

(b)

Fig. 8. output

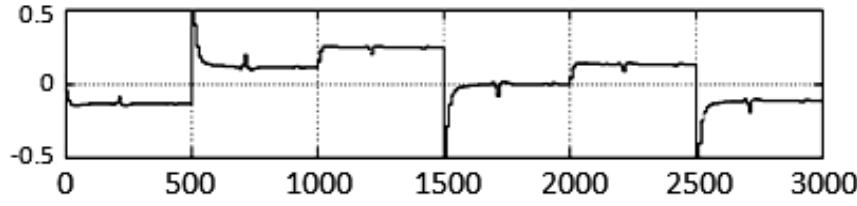

$\mathrm{M}=2, \mathrm{~N}=20, \mathrm{P}=3, \alpha=0.5, \mathrm{~W} 2=0$.

(a)

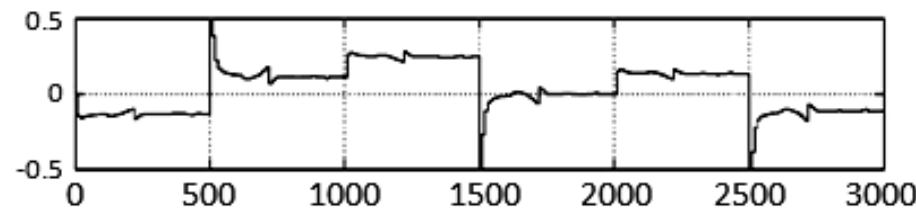

$\mathrm{M}=2, \mathrm{~N}=20, \mathrm{P}=10, \alpha=0.5, \mathrm{~W} 2=0$.

(b)

Fig. 9. control law.

\section{Effect of $W 2$}

Figures 10, 11 show the effect of changing weight factor W2 (See Eq. 5) on the system output and the control law. It is seen that by increasing the value of W2 increases the settling time while the control effort is decreased and computational complexity is not changed.

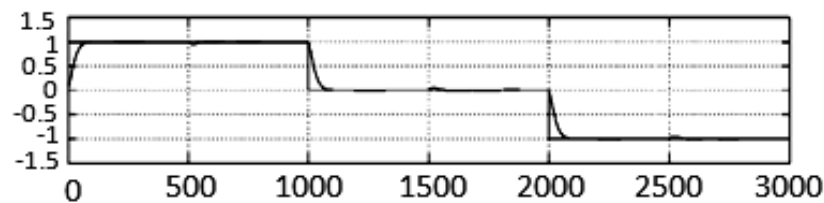

$\mathrm{M}=2, \mathrm{~N}=20, \mathrm{P}=5, \alpha=0.5, \mathrm{~W} 2=0.1$.

(a)

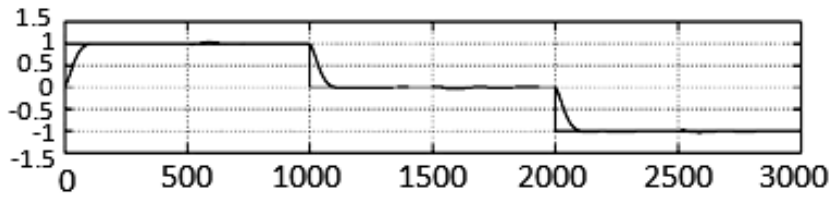

$\mathrm{M}=2, \mathrm{~N}=20, \mathrm{P}=5, \alpha=0.5, \mathrm{~W} 2=1$.

(b)

Fig.10. output. 


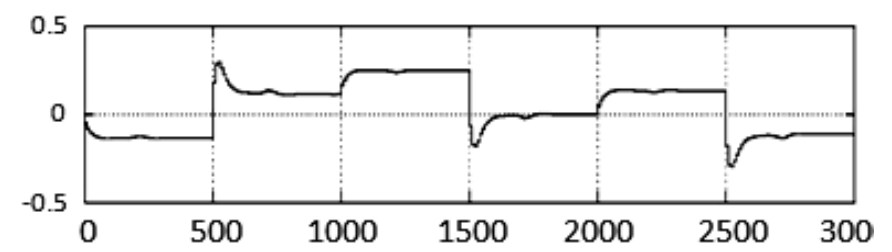

$\mathrm{M}=2, \mathrm{~N}=20, \mathrm{P}=5, \alpha=0.5, \mathrm{~W} 2=0.1$.

(a)

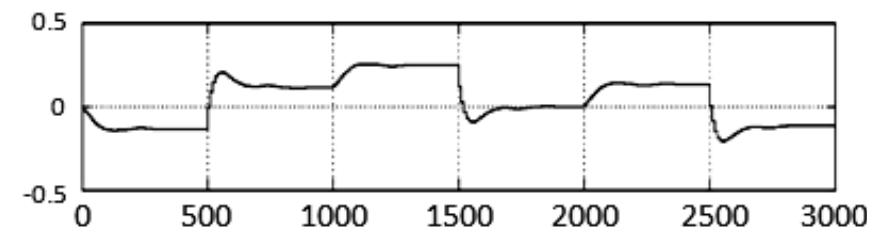

$\mathrm{M}=2, \mathrm{~N}=20, \mathrm{P}=5, \alpha=0.5, \mathrm{~W} 2=1$.

(b)

Fig.11. control law.

\section{Effect of $\alpha$}

Figures 12 and 13 show the effect of variation of $\alpha$ used in the input signal filter band and applied on the control output and control law respectively. According to these figure increasing the value of $\alpha$, increases the settling time and decreases the control effort. However the computational complexity is not changed.

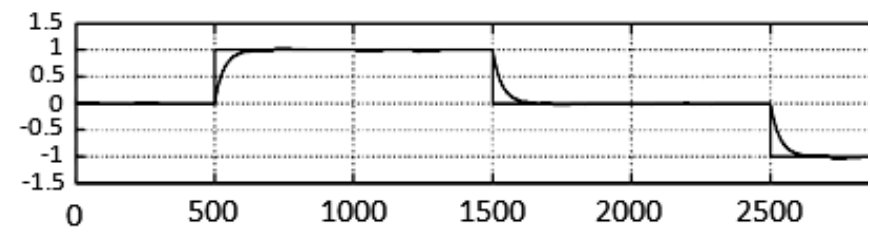

$\mathrm{M}=2, \mathrm{~N}=20, \mathrm{P}=5, \alpha=0.7, \mathrm{~W} 2=0$.

(a)

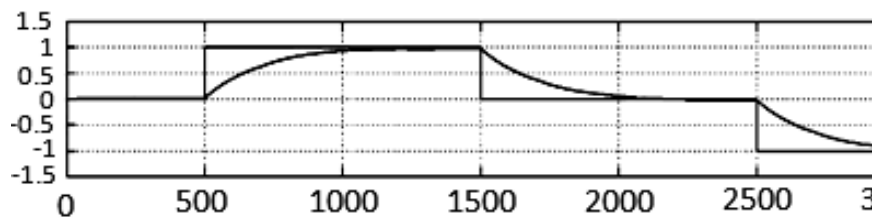
$\mathrm{M}=2, \mathrm{~N}=20, \mathrm{P}=5, \alpha=0.9, \mathrm{~W} 2=0$.

(b)

Fig. 12. Output.

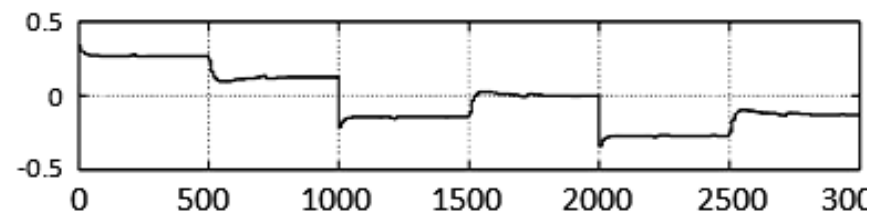

$\mathrm{M}=2, \mathrm{~N}=20, \mathrm{P}=5, \alpha=0.7, \mathrm{~W} 2=0$.

(a)

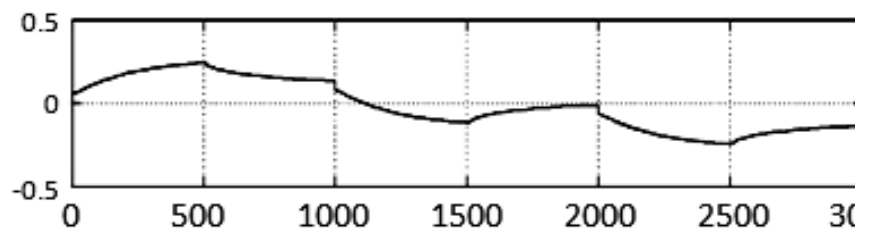

$\mathrm{M}=2, \mathrm{~N}=20, \mathrm{P}=5, \alpha=0.9, \mathrm{~W} 2=0$.

(b)

Fig.13control law.

\section{COMPARISON OF MPC WITH OTHER CONTROL MODELS}

To show the effectiveness of the MPC controller three different controllers (MPC, PID and adaptive) are implemented on P2AT mobile robot and the system is tested in an elliptical path (Fig. 14).

PID control tuning is described at (Gu et al,1997)

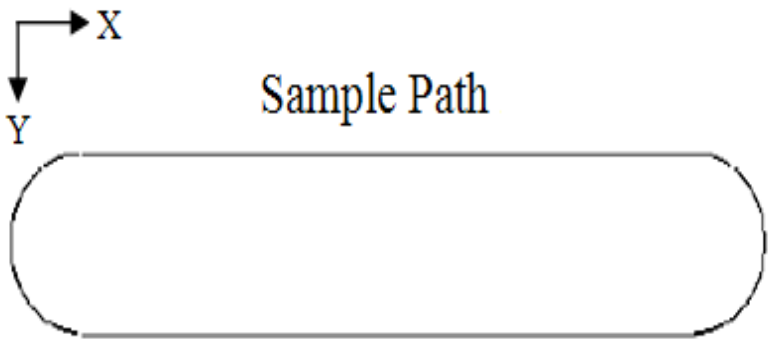

Fig.14.elliptical path.

Figures 15-17 show the error and its first derivative for different controllers. Moreover the real path moved by the robot is given in the subplots. As figure 17 shows, the MPC controller has a lower error compared to the other control methods and can track the path more precisely.

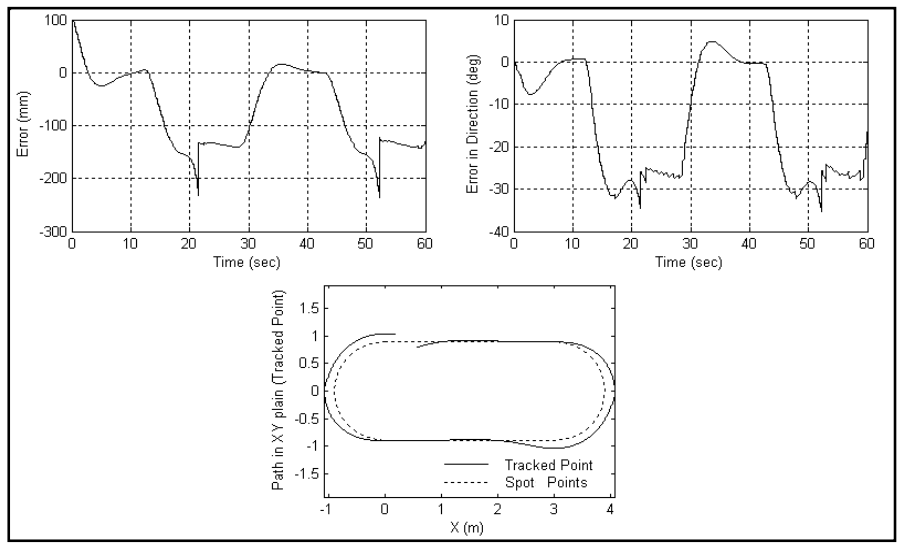

Fig.15.Robot path with PID controller. 


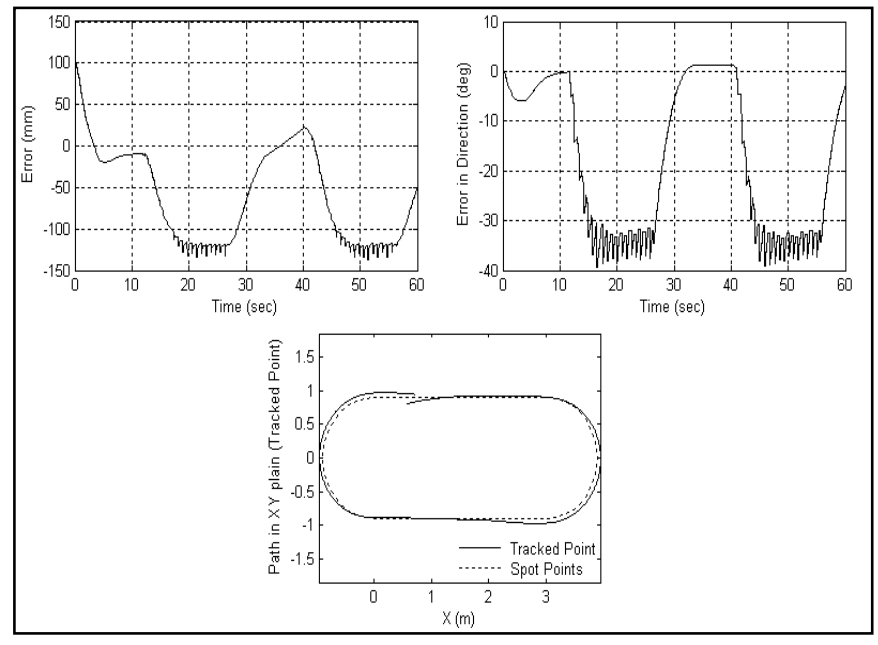

Fig. 16. Robot path with adaptive controller.

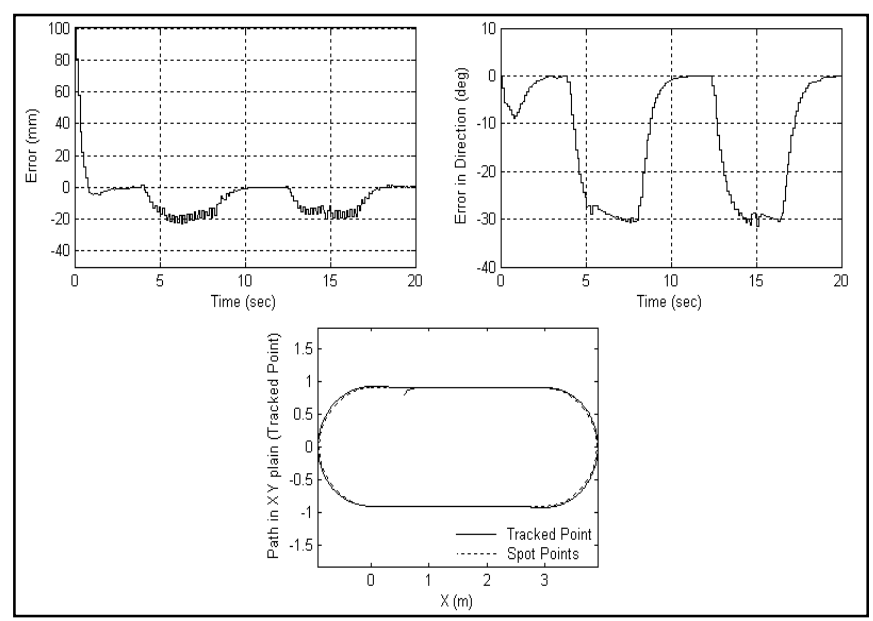

Fig.17. Robot path with MPC controller.

\section{CONCLUSION}

In this paper implementation of MPC controller on P2AT robot was explained. The conducted experiments show effectiveness of the proposed method on control of the mobile robot. Furthermore the effects of the model parameters such as control horizon, prediction horizon, weighting factor and signal filter band on the controller performance were studied. Finally, a comparison between the designed MPC controller and PID and adaptive controllers was presented demonstrating superior performance of the Model Predictive Controllers.

\section{REFERENCES}

[1] Guang, Li., Lennox, B., Zhengtao, Ding., 2005, Infinite horizon model predictive control for tracking problems,

[2] Control and Automation,. ICCA '05. International Conference on, pages $516-521$.

[3] Camacho, E.F., Bordons, C., 1999. Model Predictive Control, SpringerVerlag 2 edition.

[4] Nagy, Z. , Franke, R., Mahn, B., Allg , ower, F., 2005, Real-time implementation of nonlinear model predictive control of inite time processes in an industrial,framework. In International Workshop on Assessment and Future Directions of Nonlinear Model Predictive Control, Germany, pages 483-490.

[5] Likar, B., Kocijan, J., 2007, Predictive control of a gas-liquid separation plant based on a Gaussian process model. Computers \& Chemical Engineering, 31(1):142-152.

[6] Hauge, T.A., Slora, R. and Lie, B., Model Predictive Control of a Norske Skog, Preliminary Study, in proceedings of Control Systems,2002, June 3-5, Stockholm, Sweden, pages 75-79.

[7] Garcia, C.E., Prett, D.M, Morari, M., 1989. Model Predictive Control: Theory and Practice, A Survey. Automatica, 25(3):335-348.

[8] Limon, D., Álamo, T., Camacho, E.F., 2005. Enlarging the domain of attraction of MPC controllers, Automatica.

[9] Kouvaritakis, B., Cannon, M., Couchman, P., 2006, MPC as a tool for sustainable development integrated policy assessment. IEEE Transactions on Automatic Control, 51(145-149).

[10] Bellemans, B., Schutter, D., De Moor, B., 2006, Model predictive control for ramp metering of motorway traffic: A case study, Control Engineering Practice, 14(7): 757-767.

[11] van den Boom, T.J.J., De Schutter, B., 2006. MPC of implicit switching max-plus-linear discrete event systems - Timing aspects, Proceedings of the 8th International Workshop on Discrete Event Systems (WODES'06), Ann Arbor, Michigan, pages 457-462.

[12] Azevedo, C., Poignet, P., Espiau, B., 2002. Moving horizon control for biped robots without reference trajectory. In IEEE International Conference on Robotics and Automation, pages 2762-2767.

[13] Shridhar, R., Cooper, J., 1997. A Tuning Strategy for Unconstrained SISO Model Predictive Control. England: Chem..

[14] Mayne, D.Q., Rawlings, J.B, Rao, C.V., Scokaert, P., 2000. Constrained model predictive control: Stability and optimality. Automatica, 36:789814.

[15] Axehill D., 2004. A Preprocessing Algorithm with Applications to MPC, 43th IEEE Conference on Decision and Control.

[16] Axehila, D., 2004. A Preprocessing Algorithm with Applications to MPC , Fifth Conference on Computer Science and Systems Engineering, Norrköping, Sweden, October 21.

[17] Dougherty D., Cooper, D., 2004. A practical multiple model adaptive strategy for single- loop MPC.

[18] Gilbert, E. G., Tan., 1991. K., Linear system with state and control constraints: The theory and application of maximal output admissible sets. IEEE Transactions on Automatic Control, 36:1008-1020.

[18]D. Gu, H. Hu, M. Brady, F. Li,1997, Navigation System for Autonomous Mobile Robots at Oxford, Proceedings of International Workshop on Recent Advances in Mobile Robots, Leicester, U.K., , 1-2: 24-33.

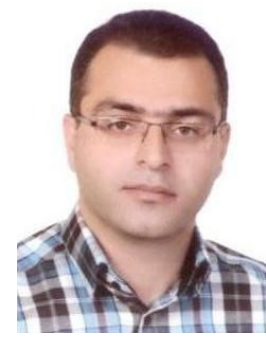

Alireza Rezaee received his B.Sc. degree in Control Engineering from Sharif University of Technology, Iran 2002 and M.Sc and Ph.D degree in Electrical Engineering from Amirkabir University of Technology, Iran (2005 and 2011 respectively). From 2013 till now he is an assistance professor in department of system and mechatronics engineering in new sciences and technology faculty at university of Tehran. His research interest are in intelligent robotics, mobile robot, navigation, machine learning, bayesian networks, cognitive science 